\title{
Two-dimensional turbulent convection
}

Andrea Mazzino

Citation: Physics of Fluids 29, 111102 (2017);

View online: https://doi.org/10.1063/1.4990083

View Table of Contents: http://aip.scitation.org/toc/phf/29/11

Published by the American Institute of Physics

\section{Articles you may be interested in}

From two-dimensional to three-dimensional turbulence through two-dimensional three-component flows Physics of Fluids 29, 111101 (2017); 10.1063/1.4990082

Dissipation of coherent structures in confined two-dimensional turbulence

Physics of Fluids 29, 111103 (2017); 10.1063/1.4993488

Two-dimensional turbulence in three-dimensional flows

Physics of Fluids 29, 111107 (2017); 10.1063/1.5000863

Vortex scaling ranges in two-dimensional turbulence

Physics of Fluids 29, 111104 (2017); 10.1063/1.4993144

Multiple stages of decay in two-dimensional turbulence

Physics of Fluids 29, 111105 (2017); 10.1063/1.4996776

Introduction to Focus Issue: Two-Dimensional Turbulence

Physics of Fluids 29, 110901 (2017); 10.1063/1.5012997

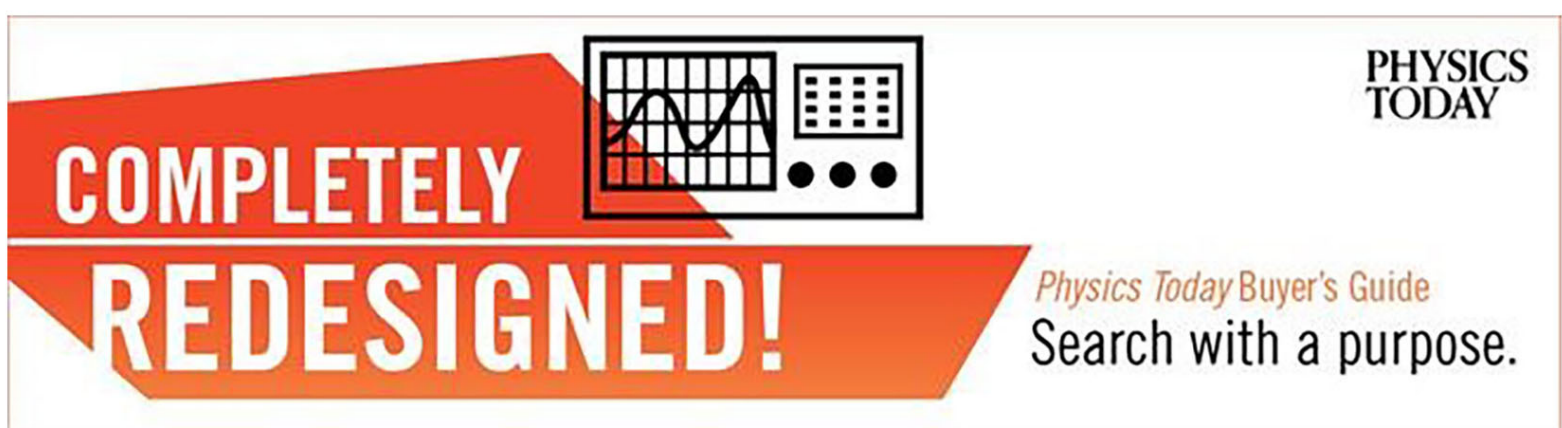




\title{
Two-dimensional turbulent convection
}

\author{
Andrea Mazzino \\ DICCA, Università di Genova, Via Montallegro 1, I-16145 Genova, Italy; Consorzio CINFAI, Via Montallegro 1, \\ I-16145 Genova, Italy; and Istituto Nazionale di Fisica Nucleare - Sezione di Genova, Via Dodecaneso 33, \\ I-16146 Genova, Italy
}

(Received 12 April 2017; accepted 31 May 2017; published online 7 July 2017)

\begin{abstract}
We present an overview of the most relevant, and sometimes contrasting, theoretical approaches to Rayleigh-Taylor and mean-gradient-forced Rayleigh-Bénard two-dimensional turbulence together with numerical and experimental evidences for their support. The main aim of this overview is to emphasize that, despite the different character of these two systems, especially in relation to their steadiness/unsteadiness, turbulent fluctuations are well described by the same scaling relationships originated from the Bolgiano balance. The latter states that inertial terms and buoyancy terms balance at small scales giving rise to an inverse kinetic energy cascade. The main difference with respect to the inverse energy cascade in hydrodynamic turbulence [R. H. Kraichnan, "Inertial ranges in twodimensional turbulence," Phys. Fluids 10, 1417 (1967)] is that the rate of cascade of kinetic energy here is not constant along the inertial range of scales. Thanks to the absence of physical boundaries, the two systems here investigated turned out to be a natural physical realization of the Kraichnan scaling regime hitherto associated with the elusive "ultimate state of thermal convection" [R. H. Kraichnan, "Turbulent thermal convection at arbitrary Prandtl number," Phys. Fluids 5, 1374-1389 (1962)]. Published by AIP Publishing. [http://dx.doi.org/10.1063/1.4990083]
\end{abstract}

\section{INTRODUCTION}

Thermal convection at very high Rayleigh numbers is a subject of great importance in a variety of situations ranging from geophysics to astrophysics with important consequences on many technological problems. ${ }^{1-4}$ Plumes are wellorganized structures of warm rising cold descending air that appear when the buoyancy effects are important. In the atmosphere, their generation and interaction strongly affect the heat transport properties and the temperature statistics in the convective boundary layer. ${ }^{5-8}$

Despite the fact that turbulent convection is intimately a three-dimensional phenomenon, many studies in the past have focused attention on the two-dimensional case. The results presented in Ref. 9 show that two-dimensional turbulent convection and its three-dimensional counterpart are actually dynamically connected. By analyzing the RayleighTaylor system via high-resolution direct numerical simulations (DNSs), it has been found in Ref. 9 that the system undergoes a transition from a three- to two-dimensional turbulent regime when the width of the turbulent mixing layer becomes larger than the scale of confinement. The latter scale is associated with a transverse side (along the horizontal direction) of the domain which is much smaller than the others. In the late stage of the evolution, the convective flow is characterized by the coexistence of Kolmogorov-Obukhov (characterizing fully three-dimensional cases) and Bolgiano-Obukhov (characterizing fully two-dimensional cases) scaling at small and large scales, respectively.

Two-dimensional turbulent convection and their peculiar properties are thus an important playground not only per sé but also to achieve a better understanding of the three-dimensional problem.
A fundamental issue in turbulent convection is the determination of the statistical properties of velocity and temperature fluctuations in the inertial range of scales in which turbulent mixing is at work. The present paper aims at summarizing the main results in this context for the two-dimensional case. The focus will be on two paradigmatic systems of turbulent convection, the Rayleigh-Bénard (RB) and the RayleighTaylor (RT) systems. As we will see, important quantitative properties related to the scaling of relevant statistical observables in the inertial range of scales are shared by the two systems.

The paper is organized as follows. In Sec. I, we introduce the equations of motion and we provide the theoretical background. In Sec. III, we discuss different predictions based on phenomenological theories for two-point statistical observables. Numerical results corroborating the predictions will be also reported and discussed with emphasis on the statistics of extreme fluctuations leading to intermittency saturation. In Sec. IV, the scaling of global observables is discussed in relation to the famous "ultimate state of thermal convection." Conclusions and perspectives are reported in the last section.

\section{RULING EQUATIONS}

The two-dimensional equations of thermal convection ruling the evolution of flow velocity and temperature in the Oberbeck-Boussinesq approximation are

$$
\begin{aligned}
\partial_{t} T+\boldsymbol{v} \cdot \boldsymbol{\partial} T & =\kappa \partial^{2} T, \\
\partial_{t} \omega+\boldsymbol{v} \cdot \boldsymbol{\partial} \omega & =v \partial^{2} \omega-\beta \boldsymbol{\partial} T \times \boldsymbol{g},
\end{aligned}
$$


T being the temperature field, $\omega=\boldsymbol{\partial} \times \boldsymbol{v}$ the vorticity, $\boldsymbol{g}$ the gravitational acceleration, $\beta$ the thermal expansion coefficient, $\kappa$ the molecular diffusivity, and $v$ the viscosity.

These equations rule the dynamics of both RB and RT systems. To distinguish the two systems, one needs to specify forcing conditions, initial and boundary conditions. For the Rayleigh-Taylor problem, at the initial time $t=0$, the system is at rest with the colder fluid placed above the hotter one (see Fig. 1 for a sketch of the late-stage evolution of the system). This amounts to assuming a step function for the initial temperature profile, $T(0, x)=-\operatorname{sgn}(z) \Theta / 2, \Theta$ being the initial temperature jump. The lateral boundary conditions are periodic, whereas along the vertical, the box is assumed to be, ideally, of infinite extension.

For the Rayleigh-Bénard problem, since the fluid is being heated from below, a linear profile $\boldsymbol{G} \cdot \boldsymbol{x}$ is assumed for the horizontally averaged temperature profile, with a large-scale gradient $\boldsymbol{G}$ pointing downward as the gravity field. The boundary conditions for the fluctuations around the horizontally averaged temperature are bi-periodic (along both directions). In a similar model, studied in Refs. 11 and 12, no mean gradient is present and a forcing term is added to the equation for the temperature field. That model mimics the convective layer far from the physical boundaries.

In both cases discussed, for RT and RB systems considered here the boundary conditions are such to avoid

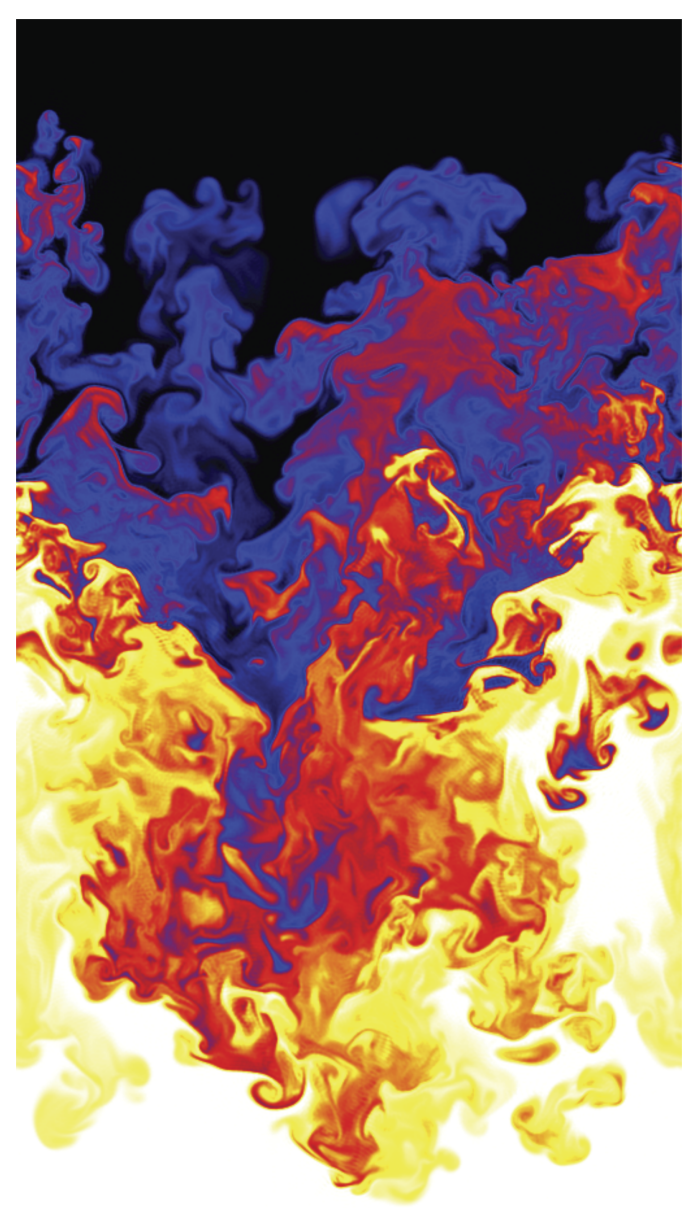

FIG. 1. Image of the temperature field in RT turbulence obtained from a DNS with the same parameters reported in Ref. 10. Black is the cold flow, whereas white is the warm flow. Gravity points downwards. the formation of boundary layers. There are however interesting studies where boundaries are explicitly taken into account, both at the level of numerical simulations (see, e.g., Refs. 13 and 14) and experiments (see, e.g., Refs. 13, 15, and 16).

In Eq. (2), the temperature field affects the vorticity through the buoyancy forces, thus providing a simple example of active scalar turbulence. At large enough values of $\beta$, the buoyancy forces might equilibrate the inertial terms in the velocity dynamics, while the temperature fluctuations cascade toward the small scales. However, it is also a priori possible in the opposite scenario with the buoyancy contribution remaining confined to the sole large scales. Different scaling behaviors in the inertial range of scales are expected in these two, totally different, regimes. Understanding the actual scaling regime in two-dimensional turbulent convection is the aim of Sec. III.

\section{SCALING OF TWO-POINT OBSERVABLES}

Two different predictions for scaling regimes associated with the dynamics ruled by Eq. (2) can be formulated. Let us start from the first. Upon assuming that the temperature behaves as a passive scalar, the analysis of two-dimensional Navier-Stokes turbulence leads to two scenarios. While temperature variance flows to small scales at a constant flux, the velocity field either undergoes an inverse cascade with an inertial range characterized by a backward scale-independent energy flux or it develops a direct enstrophy cascade (for background information on two-dimensional turbulence, see Ref. 17 for a theoretical introduction and Refs. 18-20 for more recent reviews). For the RT case, it has been shown in Ref. 21 that both possibilities actually turn out to be inconsistent. As we will see in the following, numerical results support the fact that the same conclusion holds true also for the RB system.

An alternative prediction, proposed by Bolgiano ${ }^{22}$ and Obukhov $^{23}$ in discussing the statistics of velocity and temperature fluctuations in a stably stratified atmosphere, can be formulated for our systems. According to their assumptions, the buoyancy forces allow the introduction of a characteristic scale, the Bolgiano scale $L_{B}$, above which buoyancy becomes important and the statistics of the velocity and temperature is determined by the balance between buoyancy and inertia forces. This leads to the Bolgiano-Obukhov scaling $(\mathrm{BO})^{2}$ for the velocity and temperature structure functions.

These latter statistical indicators are defined as

$$
\begin{aligned}
& S_{p}(r)=\left\langle\left[(\boldsymbol{v}(\boldsymbol{r}, t)-\boldsymbol{v}(\mathbf{0}, t)) \cdot \frac{\boldsymbol{r}}{r}\right]^{p}\right\rangle, \\
& S_{p}^{T}(r)=\left\langle[T(\boldsymbol{r}, t)-T(\mathbf{0}, t)]^{p}\right\rangle .
\end{aligned}
$$

In the above expressions, brackets denote space averages within the mixing layer under the hypothesis of small-scale homogeneity and isotropy. The BO scaling is equivalent to the following (dimensional) scaling relations: $S_{p}(r) \simeq A_{p} r^{3 p / 5}$ and $S_{p}^{T}(r) \simeq B_{p} r^{p / 5}$. 
This dependence on the scale separation is the same for both RT and RB turbulence. Differences only appear in the prefactors $A_{p}$ and $B_{p}$. In RB turbulence, they do not depend on time whereas they do so for RT turbulence, the fingerprint of the intimately unsteady nature of RT turbulence. The fact that the spatial scaling for RT and RB is the same is the result of the key assumption done by Chertkov in Ref. 21: the cornerstone of his theory is indeed the quasi-equilibrium picture where small scales adjust adiabatically as temperature and velocity fluctuations decay in time. Following this picture, one arrives at $A_{p}=(\beta g \Theta)^{2 / 5} t^{-1 / 5}$ and $B_{p}=(\beta g)^{-1 / 5} \Theta^{4 / 5} t^{-2 / 5}$.

These results constitute a set of mean field (i.e., dimensional) predictions, which need to be verified against numerical simulations and/or experiments. This fact is discussed in Sec. III A.

\section{A. Numerical results}

We review here the available results showing at which extent the BO regime is encountered for RB and RT turbulence. Let us start by analyzing the former system. We refer to Refs. 24 and 25 for all details, including the numerical setting of the exploited DNS. Figure 2 shows the behavior of scaling exponents of both temperature structure functions, $\zeta_{p}^{T}$, and velocity structure functions, $\zeta_{p}$. One can easily observe that while velocity fluctuations closely follow the BO dimensional predictions, this is not the case for temperature fluctuations. These temperature fluctuations deviate from the power-law $r^{p / 5}$ - a fingerprint of intermittency - already at the level of fourth-order structure functions. Intermittency increases with the order $p$ and tends to saturate at a constant value $\zeta_{\infty} \sim 0.8$. From this figure, one can easily see that saturation is achieved from $p \sim 8$. The absence of intermittency for velocity fluctuations is accompanied with the emergence of an inverse energy cascade (see inset of Fig. 10 in Ref. 9) which, unlike what happens in two-dimensional hydrodynamic turbulence, ${ }^{26}$ blows to large scales with a non-constant flux.

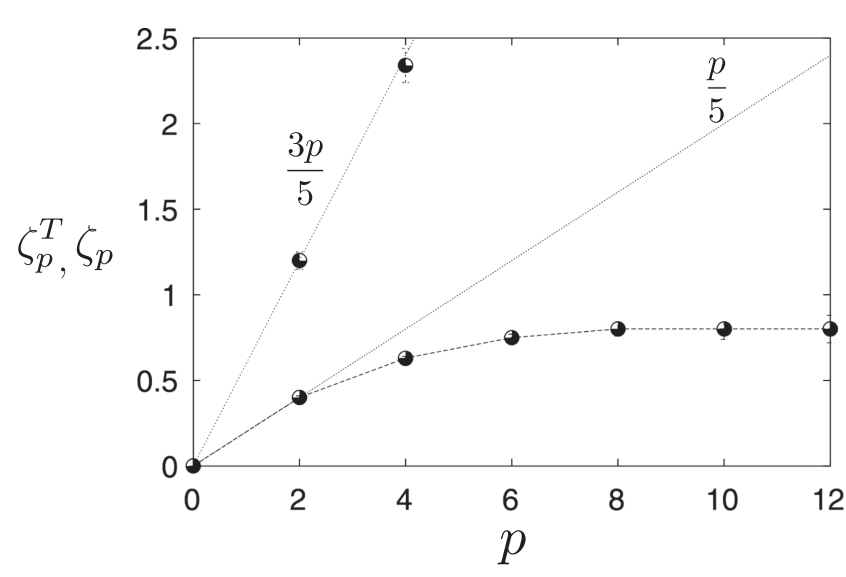

FIG. 2. Scaling exponents of temperature increments, $\zeta_{p}^{T}$, and velocity increments, $\zeta_{p}$, for the RB system. The straight lines denote the dimensional predictions: $p / 5$ for temperature and $3 p / 5$ for velocity. Notice that at orders larger than $p=8$, the temperature exponents saturate to a constant value, $\zeta_{\infty} \sim 0.8$. Details of the numerics can be found in Ref. 24. Adapted with permission from A. Celani, A. Mazzino, and M. Vergassola, "Thermal plume turbulence," Phys. Fluids 13, 2133-2135 (2001). Copyright 2001 AIP Publishing LLC.
Let us now pass to analyze scaling exponents for the RT system. They have been obtained in Ref. 10 and, more recently, in Refs. 27 and 28. The results are reported in Fig. 3 which shows the behavior of velocity and temperature structure functions up to the order $p=6$. Also in this case, within error bars (corresponding to relative errors of about $10 \%$ ), velocity fluctuations do not show intermittency, whereas temperature displays scaling exponents compatible with those of Fig. 2 relative to the RB system. To understand the reason at the origin of the possible equivalence between scaling exponents of RT and RB systems, it is sufficient to look at the horizontally averaged temperature field. This observable is depicted in Fig. 4. It is worth noticing the almost linear behavior of the averaged temperature within the mixed layer. The linearity of the mean temperature profile implies statistical homogeneity inside the mixing layer, a key ingredient for the development of the phenomenological theory à la Kolmogorov used in Ref. 21 . We can thus conclude that fluctuations in RT turbulence are forced, as in RB turbulence, by a linear mean temperature profile. In RB turbulence, it is assigned in the form of external
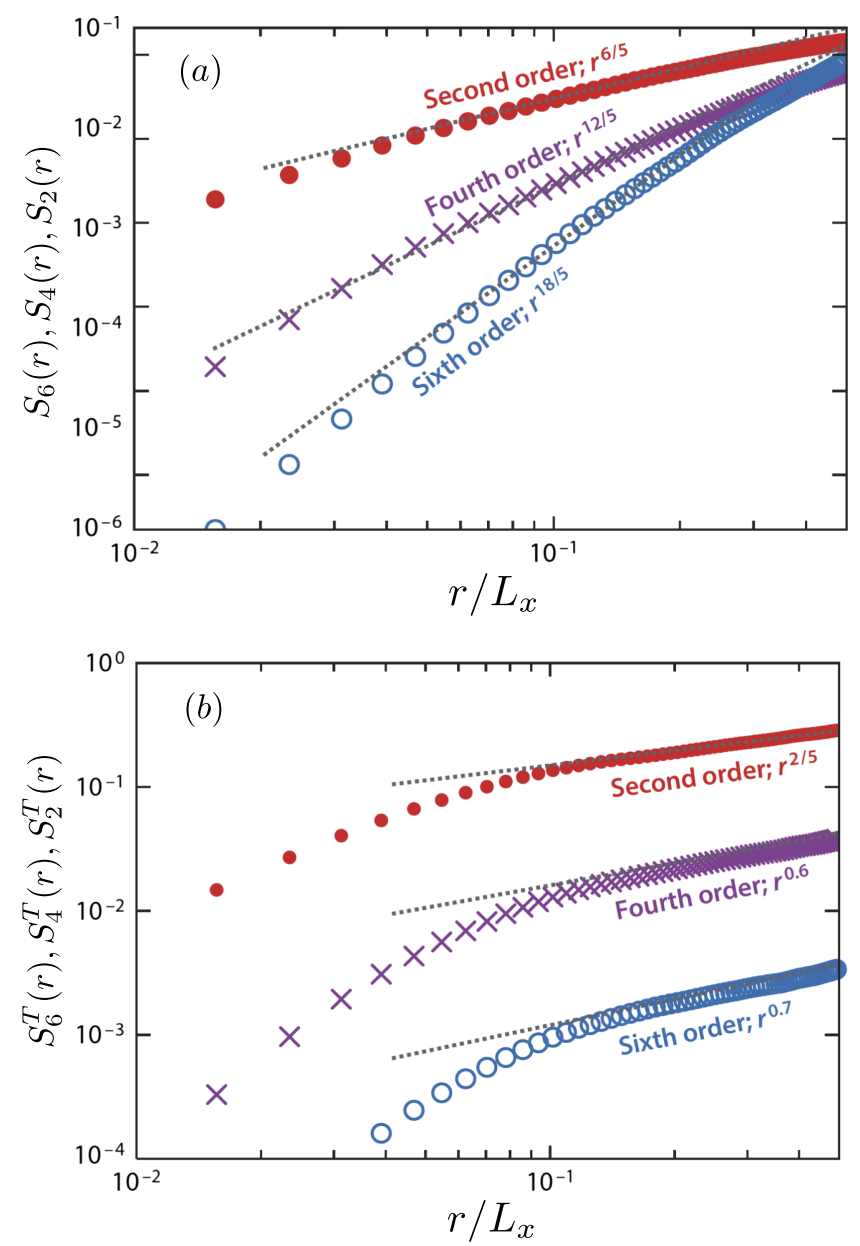

FIG. 3. Isotropic moments for the RT system of the longitudinal (a) velocity differences and (b) temperature differences of orders 2, 4, and 6 obtained by averaging over all directions of separation $\mathrm{r}$. In panel (a), the gray dotted lines represent the Bolgiano dimensional prediction $r^{3 p / 5}$, whereas in panel (b), they represent the best-fit scaling exponents, which for $p=4$ and $p=6$ are anomalous. $L_{x}$ denotes the horizontal size of the computational domain. Adapted with permission from A. Mazzino and G. Boffetta, "Incompressible Rayleigh-Taylor turbulence,” Annu. Rev. Fluid Mech. 49, 119143 (2017). Copyright 2017 Annual Reviews. 


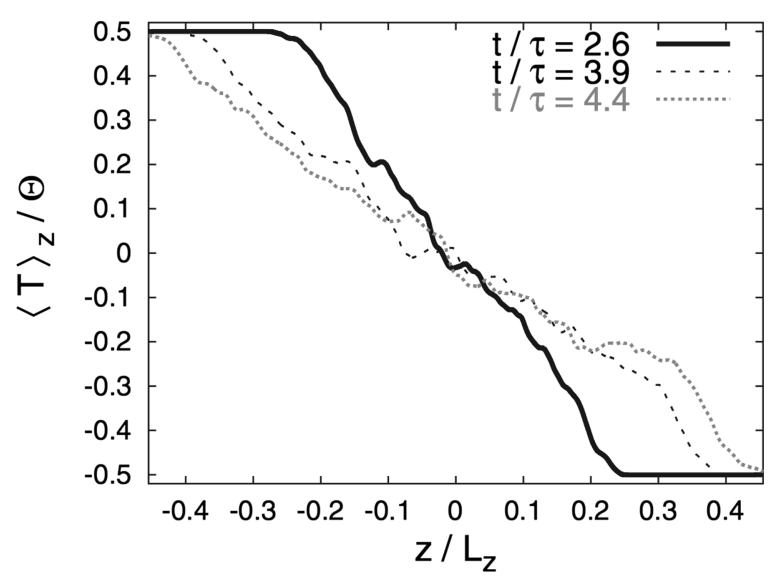

FIG. 4. The horizontally ensemble-averaged temperature field at different instants for the RT system. The dimensional group $\tau \equiv\left(2 L_{z} /(\beta \Theta g)\right)^{1 / 2}$ is a characteristic time scale of the flow. Here, $L_{z}$ is the vertical size of the box. Note the almost linear behavior of $\langle T\rangle_{z}$ in the mixed layer. Adapted with permission from A. Celani, A. Mazzino, and L. Vozella, "Rayleigh-Taylor turbulence in two dimensions," Phys. Rev. Lett. 96, 134504 (2006). Copyright 2006 American Physical Society.

forcing, whereas in RT turbulence, it is created by the intrinsic dynamics. The fact that RT turbulence is a non-stationary problem does not matter here because of the quasi-steady scenario argued by Chertkov in Ref. 21. The evidences reported here provide a strong support to Chertkov's hypothesis on the quasiequilibrium between large-scale and small-scale fluctuations in RT turbulence. From a more general point of view, they also point to the conclusion that RT and RB belong to the same class of universality with respect to boundary conditions/forcing mechanisms.

\section{B. A link between geometry and statistics}

If one looks at Fig. 1, it appears that plumes are formed by a well-mixed (hot or cold) region, where temperature fluctuations are weak, bounded by a thin interface separating warm (cold) fluid from the colder (warmer) surrounding background. Separations $r$ between points crossing the interface thus capture the largest temperature excursions, $\delta_{r} T \gg T_{r m s}$, $T_{r m s}$ being the temperature root mean square. Temperature differences across a plume interface are thus expected to contribute to the tail of the probability density function (pdf), $P$, of $\delta_{r} T$. Moreover, interfaces separating hot plumes from cold plumes appear in the form of wrinkled lines. Interestingly, the geometrical properties of the set defined by plume interfaces are linked to the extreme events of temperature fluctuations. Let us address this point in detail.

From the results shown in Ref. 24 (Fig. 2) for RB turbulence, the tails of the pdfs, $P\left(\delta_{r} T\right)$, at various separations $r$ within the inertial range of scales can be collapsed onto a single curve by a simple multiplicative factor $r^{\zeta_{\infty}}$ with $\zeta_{\infty} \sim 0.8$. This amounts to saying that $P\left(\delta_{r} T\right)=Q r^{\zeta_{\infty}}$ with the prefactor $Q$ that depends solely on the intensity of the fluctuation. Physically, this means that to select a large temperature fluctuation between points separated by a distance $r$, one first needs to intercept a plume interface, and this happens with a probability $\propto r^{\zeta_{\infty}}$, and then to have a large temperature excursion, a fact that occurs with probability $Q$. The link between geometry and statistics immediately follows from the very definition of fractal dimension, $D_{F}$, of the set hosting plume interfaces. In terms of $D_{F}$, one easily finds that the probability to intercept an interface across $\boldsymbol{r}$ goes as $r^{2-D_{F}}$. This last relationship provides the link we are searching for $D_{F}=2-\zeta_{\infty}$. The value $D_{F}=0.2$ has been obtained in Ref. 24 by the standard box-counting technique, a fact that also explains the visual impression in Fig. 1 of the interfaces as wrinkled lines.

Although our attention has been focused on results obtained for the RT system, all can be easily rephrased for the RB system too.

\section{SCALING OF GLOBAL OBSERVABLES}

Heat transfer in RT turbulence is inherently associated with the presence of turbulence. The same holds true for the RB system forced by the mean gradient. It is however worth stressing that, conversely, heat transfer in classical RB convection with boundaries is dominated by the physics at the boundary layers (both thermal and kinetic), which develop in correspondence with the two plates. As we will see, it is just the absence of boundary layers in our models for RT and RB turbulence which will allow one to isolate a scaling regime, known as the ultimate state of thermal convection, which remained elusive in RB convection with physical boundaries, even at the largest Rayleigh numbers achieved.

A way to quantify the efficiency of heat transfer due to the mixing induced by turbulence can be in terms of the Nusselt number, $\mathrm{Nu}$, the ratio of the total heat transfer to the molecular one. Other key dimensionless parameters are the Reynolds number, $R e$, a measure of turbulence intensity; the Rayleigh number, $R a$, a measure of the temperature variation along the vertical; and the Prandtl number, $P r$, the ratio between kinematic viscosity and thermal diffusivity.

A key question in turbulent convection is on the understanding of the dependence of heat transfer efficiency due to turbulence and turbulence intensity as a function of the temperature difference between upper and lower un-mixed fluids. Rephrasing the question in mathematical terms, it amounts to investigating the scaling relationship between $N u, R a$, and $R e: N u \propto R a^{\gamma}$ and $R e \propto R a^{\beta}$ with $\gamma$ and $\beta$ to be determined with prefactors which may depend on $\operatorname{Pr}$ (via power laws).

For RT turbulence, the above scaling relationships have been investigated in Refs. 10 and 27. The numerical results reported in those papers clearly show $\gamma=\beta=1 / 2$. The scaling relationships $N u \propto R a^{1 / 2}$ and $R e \propto R a^{1 / 2}$ coincide with the results derived by Kraichnan ${ }^{33}$ for the pure bulk contribution to 3D RB turbulence, also known as "the ultimate state of thermal convection," which however has eluded both experimental and numerical confirmation. ${ }^{29}$ Recent experiments for RB turbulence done in vertical channels in the absence of plates have shown indeed the appearance of a regime compatible with the ultimate state..$^{30,31}$ The situation seems however still open in RB turbulence. ${ }^{32}$

Let us provide simple arguments through which the scaling laws associated with the ultimate state of thermal convection can be derived dimensionally starting from the energy 


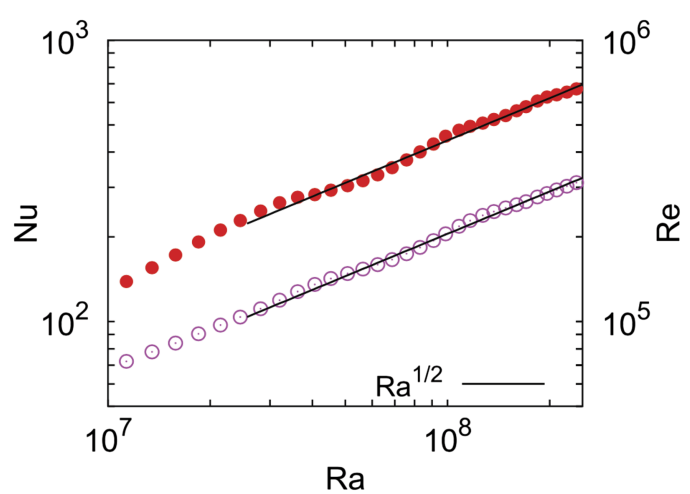

FIG. 5. The behavior of the Nusselt number (ordinate axis on the left) and Reynolds number (ordinate axis on the right) versus the Rayleigh number obtained from the results of DNSs reported in Ref. 10. Adapted with permission from A. Celani, A. Mazzino, and L. Vozella, "Rayleigh-Taylor turbulence in two dimensions," Phys. Rev. Lett. 96, 134504 (2006). Copyright 2006 American Physical Society.

equation. The latter simply follows from evolution equations (1) and (2). Neglecting the contribution of thermal diffusivity as it is smaller than the contribution from turbulence, the kinetic energy equation reads

$$
\frac{d E}{d t}=\beta g\left\langle v_{z} T\right\rangle-\epsilon_{v}=-\frac{d P}{d t}-\epsilon_{\nu},
$$

where $E=(1 / 2)\left\langle|\boldsymbol{v}|^{2}\right\rangle$ is the kinetic energy density, $P$ $=-\beta g\langle z T\rangle$ is the potential energy of the system, and $\epsilon_{v}$ $=v\left\langle|\partial v|^{2}\right\rangle$ is the viscous energy dissipation rate. Brackets denote space averages.

Let us focus for the sake of example on the RT system. In terms of $R a=\beta g \Theta h^{3} /(v \kappa), R e=v_{r m s} h / v$, and $N u=\left\langle v_{z} T\right\rangle h /(\kappa \Theta)$, the above energy equation becomes

$$
\kappa \frac{\beta g \Theta}{h} N u=\frac{d}{d t} \frac{1}{2}\left\langle v^{2}\right\rangle+\epsilon_{v} .
$$

Here $v_{r m s}$ is the root mean square of velocity fluctuations within the mixed layer and $h(t)$ is the mixed layer width. Because of the fact that for RT turbulence we already discussed that kinetic energy blows toward large scales, one can safely neglect $\epsilon_{v}$ in (6). Together with the fact that $v_{r m s} \simeq \beta g \Theta t$ and $h(t) \simeq \beta \Theta g t^{2}$, the following dimensional predictions for $\mathrm{Nu}, \mathrm{Ra}$, and $\mathrm{Re}$ are obtained:

$$
N u=\frac{(\beta g \Theta)^{2}}{\kappa} t^{3}, R a=\frac{(\beta g \Theta)^{4}}{v \kappa} t^{6}, R e=\frac{(\beta g \Theta)^{2}}{v} t^{3} .
$$

Eliminating $t$ from the above expressions, one easily gets the scaling relationships associated with the ultimate state of thermal convection

$$
N u \simeq R a^{1 / 2} \operatorname{Pr}^{1 / 2}, \quad R e \simeq R a^{1 / 2} \operatorname{Pr}^{-1 / 2}
$$

The accuracy of these predictions in RT turbulence can be detected from Fig. 5. Data shown in this figure are obtained from Ref. 10.

\section{CONCLUSIONS AND PERSPECTIVES}

We have analyzed the (steady) mean-gradient-forced Rayleigh-Bénard (RB) turbulent system and the (unsteady)
Rayleigh-Taylor (RT) turbulent system in two dimensions. These two systems provide non-trivial examples where the temperature plays the role of active scalar within the whole inertial range of scales. In this respect, the underlying physics in the two-dimensional case totally differs with respect to the analogous three-dimensional cases where it is known that temperature behaves as a passive scalar, ${ }^{34}$ the action of buoyancy being restricted to the sole large-scale of motion. The quantitative consequence of these different behaviors reflects in the emergence of different scaling laws of relevant two-point statistical observables: instead of the classical Kolmogorov-Obukhov scenario, characterizing the fully three-dimensional cases, the Bolgiano-Obukhov picture is predicted and actually confirmed by suitable high-resolution direct numerical simulations. Despite this remarkable difference between the two-dimensional and the three-dimensional cases, these two regimes are dynamically connected. Indeed, it has been found for the case of RT turbulence ${ }^{9}$ that the system undergoes a transition from a three- to twodimensional turbulent regime when the width of the turbulent mixing layer becomes larger than the scale of confinement associated with the shortest side of the domain along the horizontal direction. In the late stage of the evolution, the convective flow is characterized by the coexistence of Kolmogorov-Obukhov and Bolgiano-Obukhov scaling.

Both RT and RB systems show absence of intermittency for velocity fluctuations. Moreover, the kinetic energy blows toward the large scales of motion. In this respect, both systems resemble the classical two-dimensional hydrodynamic turbulence in the inverse energy cascade with the major difference that for RT and RB turbulence the rate of cascade of kinetic energy is not constant along the inertial range of scales.

Intermittency is on the contrary present in the statistics of temperature fluctuations. Numerical results on RB and RT turbulence point to the conclusion that scaling exponents of temperature structure functions are the same and tend to saturate to a constant value for sufficiently large order. The concept of intermittency saturation provides a link between geometrical properties (of plume interfaces) and statistical properties (of extreme temperature excursions).

All the above discussions concern RT and RB turbulence of miscible fluids. Many interesting new issues arise in the same context but in the presence of immiscible fluids. In the latter case, because of surface tension, the systems possess new degrees of freedom (associated to capillary waves) which can be excited by hydrodynamic degrees of freedom thus interacting with turbulent fluctuations. For the three-dimensional RT turbulence of immiscible fluids, a phenomenological theory has been proposed in Ref. 35 and it predicts that the energy is carried towards small scales by both inertial and wave cascades simultaneously. A phenomenological theory in the two-dimensional case is still missing and it is not clear in which way (and if any) the inverse energy cascade can interact with the propagation of capillary waves on plume interfaces. Addressing this issue, exploiting numerical strategies like the one reported in Ref. 36 seems a doable option. 


\section{ACKNOWLEDGMENTS}

We acknowledge the financial support from PRIN 2012 (Project No. D38C13000610001 funded by the Italian Ministry of Education) and the Italian flagship project RITMARE. A fruitful collaboration with G. Boffetta, A. Celani, S. Musacchio, L. Vozella, and M. Vergassola, with whom part of the results here reviewed have been collected, is warmly acknowledged.

${ }^{1}$ E. D. Siggia, "High Rayleigh number convection," Annu. Rev. Fluid Mech. 26, 137-168 (1994).

${ }^{2}$ D. Lohse and K. Xia, "Small-scale properties of turbulent Rayleigh-Bénard convection," Annu. Rev. Fluid Mech. 42, 335-364 (2010).

${ }^{3}$ A. Mazzino and G. Boffetta, "Incompressible Rayleigh-Taylor turbulence," Annu. Rev. Fluid Mech. 49, 119-143 (2017).

${ }^{4}$ G. Ahlers, S. Grossmann, and D. Lohse, "Heat transfer and large scale dynamics in turbulent Rayleigh-Bénard convection," Rev. Mod. Phys. 81, 503-537 (2009).

${ }^{5}$ M. Antonelli, A. Mazzino, and U. Rizza, "Statistics of temperature fluctuations in a buoyancy dominated boundary layer flow simulated by a large-eddy simulation model," J. Atmos. Sci. 60, 215-224 (2003).

${ }^{6} \mathrm{C}$. Rio and F. Hourdin, "A thermal plume model for the convective boundary layer: Representation of cumulus clouds," J. Atmos. Sci. 65, 407-425 (2008).

${ }^{7}$ J. Bosbach, S. Weiss, and G. Ahlers, "Plume fragmentation by bulk interactions in turbulent Rayleigh-Bénard convection," Phys. Rev. Lett. 108, 054501 (2012).

${ }^{8}$ E. P. van der Poel, R. Ostilla-Monico, R. Verzicco, S. Grossmann, and D. Lohse, "Logarithmic mean temperature profiles and their connection to plume emissions in turbulent Rayleigh-Bénard convection," Phys. Rev. Lett. 115, 154501 (2015).

${ }^{9}$ G. Boffetta, F. De Lillo, A. Mazzino, and S. Musacchio, "Bolgiano scale in confined Rayleigh-Taylor turbulence," J. Fluid Mech. 690, 426-440 (2012).

${ }^{10}$ A. Celani, A. Mazzino, and L. Vozella, "Rayleigh-Taylor turbulence in two dimensions," Phys. Rev. Lett. 96, 134504 (2006).

${ }^{11} \mathrm{~S}$. Toh and T. Matsumoto, "The dynamics of structures of T-vorticity in 2D free convection turbulence," in Proceedings of IUTAM Symposium on Geometry and Statistics of Turbulence, Hayama, November 1999 (Kluwer Academic, Dordrecht, 1999).

${ }^{12}$ D. Biskamp and E. Schwarz, "Scaling properties of turbulent convection in two-dimensional periodic systems," Europhys. Lett. 40, 637 (1997).

${ }^{13}$ F. Massaioli, R. Benzi, and S. Succi, "Exponential tails in two-dimensional Rayleigh-Bénard convection,” Europhys. Lett. 21, 305-310 (1993).

${ }^{14}$ K. Sugiyama, R. Ni, R. J. A. M. Stevens, T. S. Chan, S.-Q. Zhou, H.-D. Xi, C. Sun, S. Grossmann, K.-Q. Xia, and D. Lohse, "Flow reversals in thermally driven turbulence," Phys. Rev. Lett. 105, 034503 (2010).

${ }^{15}$ F. Seychelles, F. Ingremeau, C. Pradere, and H. Kellay, "From intermittent to nonintermittent behavior in two dimensional thermal convection in a soap bubble," Phys. Rev. Lett. 105, 264502 (2010).
${ }^{16}$ F. Seychelles, Y. Amarouchene, M. Bessa, and H. Kellay, "Thermal convection and emergence of isolated vortices in soap bubbles," Phys. Rev. Lett. 100, 144501 (2008)

${ }^{17}$ R. H. Kraichnan and D. Montgomery, "Two-dimensional turbulence," Rep. Prog. Phys. 43, 547-619 (1980).

${ }^{18} \mathrm{P}$. Tabeling, "Two-dimensional turbulence: A physicist approach," Phys. Rep. 362, 1-62 (2002).

${ }^{19} \mathrm{H}$. Kellay and W. I. Goldburg, "Two-dimensional turbulence: A review of some recent experiments," Rep. Prog. Phys. 65, 845-894 (2002).

${ }^{20}$ G. Boffetta and R. E. Ecke, "Two-dimensional turbulence," Annu. Rev. Fluid Mech. 44, 427-451 (2012).

${ }^{21}$ M. Chertkov, "Phenomenology of Rayleigh-Taylor turbulence," Phys. Rev. Lett. 91, 115001 (2003).

${ }^{22} \mathrm{R}$. Bolgiano, "Turbulent spectra in a stably stratified atmosphere," J. Geophys. Res. 64, 2226-2229, doi:10.1029/jz064i012p02226 (1959).

${ }^{23} \mathrm{~A}$. Obukhov, "Effect of Archimedean forces on the structure of the temperature field in a turbulent flow," Dokl. Akad. Nauk SSSR 125, 1246-1249 (1959).

${ }^{24}$ A. Celani, A. Mazzino, and M. Vergassola, "Thermal plume turbulence," Phys. Fluids 13, 2133-2135 (2001).

${ }^{25}$ A. Celani, M. Cencini, A. Mazzino, and M. Vergassola, "Active and passive fields face to face," New J. Phys. 6, 72 (2004).

${ }^{26}$ R. H. Kraichnan, "Inertial ranges in two-dimensional turbulence," Phys. Fluids 10, 1417 (1967).

${ }^{27}$ L. Biferale, F. Mantovani, M. Sbragaglia, A. Scagliarini, F. Toschi, and R. Tripiccione, "High resolution numerical study of Rayleigh-Taylor turbulence using a thermal lattice Boltzmann scheme," Phys. Fluids 22, 115112 (2010).

${ }^{28}$ Q. Zhou, "Temporal evolution and scaling of mixing in two-dimensional Rayleigh-Taylor turbulence,” Phys. Fluids 25, 085107 (2013).

${ }^{29}$ G. Amati, K. Koal, F. Massaioli, K. R. Sreenivasan, and R. Verzicco, "Turbulent thermal convection at high Rayleigh numbers for a Boussinesq fluid of constant Prandtl number," Phys. Fluids 17, 121701 (2005).

${ }^{30}$ M. Gibert, H. Pabiou, F. Chillà, and B. Castaing, "High-Rayleighnumber convection in a vertical channel," Phys. Rev. Lett. 96, 084501 (2006).

${ }^{31}$ M. R. Cholemari and J. H. Arakeri, "Axially homogeneous, zero mean flow buoyancy-driven turbulence in a vertical pipe," J. Fluid Mech. 621, 69-102 (2009).

${ }^{32}$ L. Skrbek and P. Urban, "Has the ultimate state of turbulent thermal convection been observed?," J. Fluid Mech. 785, 270-282 (2015).

${ }^{33}$ R. H. Kraichnan, "Turbulent thermal convection at arbitrary Prandtl number," Phys. Fluids 5, 1374-1389 (1962).

${ }^{34}$ G. Boffetta, A. Mazzino, S. Musacchio, and L. Vozella, "Kolmogorov scaling and intermittency in Rayleigh-Taylor turbulence," Phys. Rev. E 79, 065301(R) (2009).

${ }^{35}$ M. Chertkov, I. Kolokolov, and V. Lebedev, "Effects of surface tension on immiscible Rayleigh-Taylor turbulence," Phys. Rev. E 71, 055301(R) (2005).

${ }^{36}$ A. Celani, A. Mazzino, P. Muratore-Ginanneschi, and L. Vozella, "Phasefield model for the Rayleigh-Taylor instability of immiscible fluids," J. Fluid Mech. 622, 115-134 (2009). 